\title{
Calculation of Rock Compressibility by Using the Characteristics of Downstream Pressure Change in Permeability Experiment
}

\author{
Jun He, Kegang Ling*, Peng Pei, University of North Dakota; Xiao Ni, China University \\ of Petroleum \\ ${ }^{*}$ Corresponding author: Kegang Ling \\ Email: kegang.ling@engr.und.edu
}

Keywords: Pore Compressibility, Low Permeability Reservoir

\begin{abstract}
In petroleum exploration and production, knowledge of geomechanical properties of target reservoirs ensures producing hydrocarbon safely and economically, and protecting environmental friendly. Rock compressibility, one of the geomechanical properties, is an essential parameter in drilling and completion design. Because direct measurements of rock compressibility are time consuming and cost expensive, indirect measurements from other readily available experimental data are highly demanded. When direct measurements are unavailable or experimental data are unreliable due to lab and human errors, irregular core plug, and/or non-uniform deformation, obtaining rock compressibility from other methods is not only a good reference for the directly measured rock compressibility but also an important supplement to those indirect methods. In this study, a method with solid theoretical base is developed to determine rock compressibility using permeability experimental data. With that, core analysis can be more reliable and accurate. The combination of the proposed method with direct measurements can be employed to ensure the reliability of the experiment and to quantify the uncertainty resulting from lab and human errors.
\end{abstract}

\section{Introduction}

With the decline in conventional oil and gas production worldwide, petroleum exploration and production from unconventional oil and gas resources have gained great momentum throughout the world to fill the gap between ever 
increasing demand of energy and decreasing production of conventional reservoirs. Knowledge of geomechanical properties of target reservoirs ensures producing hydrocarbons from unconventional resources safely, environmental friendly, and economically. Rock compressibility is one of the key parameters in designing the drilling and completion of oil and gas wells, modeling the fluids flow in reservoir, and forecasting the well production. There are two categories of methods to obtain rock compressibility. One is direct measurement; another is indirect measurement. Direct measurement measures compressibility through uniaxial or triaxial stress experiment. Indirect measurement estimates compressibility from correlations or other measurements. The importance of rock compressibility is reflected by numerous investigations attempting to evaluate it accurately.

Carpenter and Spencer [1] measured compressibility of consolidated oil-bearing sandstones collected from East Texas oil field at reservoir conditions. In the same manner as those reported in the Carpenter and Spencer's study, Hall [2] conducted tests to measure compressibility of limestone and sandstone and developed a correlation to estimate rock compressibility through porosity. Moreover, he found that ignoring rock compressibility can lead to 30 to 40 percent overestimation of oil in place. Fatt [3] found that rock compressibility is a function of pressure and cannot be correlated to porosity. Van der Knaap [4] proved the nonlinear stress-volume relations of elastic porous media through theoretical and experimental analysis. Harville and Hawkins [5] indicated that rock compressibility of geopressured gas reservoir is higher than that of normally pressured reservoir. Greenwald and Somerton [6] measured compressibility of Berea, Bandera, and Boise sandstones. Comparison of these compressibilities to those available in the literature indicated qualitative agreement for each of the sandstone types and for their relative behavior. Furthermore, Greenwald and Somerton [7] developed a semi-empirical model to calculated rock compressibility. The required variables for their model are initial porosity, clay content, a pore shape factor, a length and aspect ratio of representative cracks in the matrix grains, the volumetric density of these cracks, and the mineralogical composition of the sample along with the elastic moduli of the minerals present. Zimmerman et al. [8] developed relations to evaluate rock compressibility from confining and pore pressures, and verified their relations through experimental measurements on Berea, Bandera, and Boise sandstones. Poston and Chen [9] determined formation compressibility and gas in place in abnormally pressured reservoirs simultaneously using material balance. Chalaturnyk and Scott [10] summarized different geomechanical test procedures and analyzed the results. Khatchikian [11] proposed a method using the Gassman equation and reservoir parameters evaluated through log 
analysis to calculate rock compressibility. Yildiz [12] predicted rock compressibility using production data. His method is the same as Poston and Chen's [9] method. Macini and Mesini [13] measured sandstone and carbonate compressibility by both static (deformation tests) and dynamics (acoustic tests) investigations. Their study showed that compressibility is not constant, but is a function of reservoir pressure. Marchina et al. [14] measured compressibility of reservoir rocks of a heavy oil field under in-situ conditions. Li et al. [15] presented a model to calculate rock compressibility using the elastic modulus and the Poisson's ratio. Suman [16] estimated rock compressibility under reservoir conditions at different depleted stages using sonic velocity derived from 4D seismic. Ghanbarnezhad Moghanloo and Javadpour [17] modelled the pressure distribution in shale samples. They addressed the dynamic pressure change at the downstream by using a semi-analytic method of characteristics (MOC) solution. The comparison of the pressure profile and history plots indicated that their MOC solutions match the simulation results.

Because direct measurement of rock compressibility is time consuming and cost expensive. Estimation of rock compressibility from other readily available experimental data, such as sonic velocity and permeability experiment, is highly demanded. In this study, we developed a method to determine the rock compressibility using permeability experimental data. The combination of the proposed method with direct measurement can be employed to ensure the reliability of the direct measurement and to quantify the uncertainty resulting from lab and human errors, irregular core plug, and/or non-uniform deformation.

\section{Methodology}

The purpose of this study is to estimate rock compressibility from permeability experiment. To better understand the principle of the permeability experiment, it is imperative to derive the diffusivity equation that is used to model fluid flow through the rock. The following assumptions are made to derive the diffusivity equation of the test fluid flow in the core: 1) the core is homogeneous, 2) the properties of the rock are constant through the test, 3) the flow in the cylindrical core is laminar, and 4) the flow in the core is isothermal. 
Fig.1 shows the test fluid flowing through a core sample during the experiment. Nitrogen gas is used as test fluid in the experiments because the permeability of tight rock is low. Gas flows from the upstream reservoir on the left-side of the core, through the core, and out of the downstream reservoir on the right-side of the core.

For the gas flow through core sample, if we consider a control volume (from $x$ to $x+\Delta x$ ), which is the volume that the gas flows in at $x$ and out at $x+\Delta x$ during a certain time period $\Delta t$, we can obtain the governing diffusivity equation for linear gas flow inside the sample by combining the mass conservation law, Darcy's law, real gas law, and the gas pseudo-pressure concept [18]:

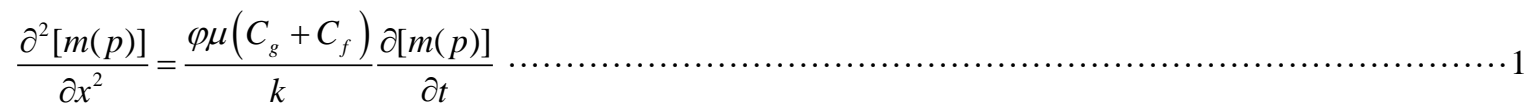

where $m(p)=\int_{p_{b}}^{p} \frac{2 p}{\mu z} d p$ is the gas pseudo-pressure, $p$ is the pore pressure inside the sample, $\varphi$ is the porosity, $C_{g}$ is the gas isothermal compressibility, $C_{f}$ is the formation compressibility, $\mu$ is the gas viscosity, and $k$ is the permeability.

In the downstream pressure build-up experiment, the sample is connected to an upstream reservoir and a downstream reservoir as shown in Fig. 2. At the beginning, the core plug is installed in a core holder, and then the core holder is pressurized by pumping mineral oil into the closed chamber. After that the test gas flows from upstream reservoir through core plug to downstream reservoir until the sample pore pressure, the upstream reservoir pressure, and the downstream reservoir pressure reach equilibrium at 14.7 psia. To allow the system to reach stabilization at a pressure of 14.7 psia, the gas infill valve needs to be closed and the inlet and out valves at both sides of the core holder need to be opened. Once the equilibrium is obtained, the inlet valve is closed and the gas infill valve is opened to pressurize the upstream reservoir to a higher level and is kept constant. Then the gas infill valve is closed and the inlet valve is opened to allow gas flow through core plug to downstream reservoir. The pressure buildup is observed in the downstream reservoir when the gas arrives. The downstream reservoir pressure keeps increasing until it reaches the value of upstream pressure. Finally the compressibility can be obtained by analyzing the characteristics of pressure change at the downstream reservoir. The equation, Eq. 14, used to estimate the compressibility is shown in the following derivation. Fig. 3 shows the upstream and downstream pressures 
recorded in an experiment in this study. According to the experiment procedure and measurement conditions, the diffusivity equation (Eq. 1) has the following boundary conditions:

(1) $m[p(x, 0)]=m\left[p_{2}(0)\right]$, for $0<x<L$. This states that at the beginning of the experiment, the sample pore pressure $(p)$ is equal to the downstream reservoir pressure $\left(p_{2}\right)$.

(2) $m[p(0, t)]=m\left[p_{1}(t)\right]$, where $p_{1}$ is the upstream reservoir pressure; for $t \geq 0$. This states that the upstream end of the sample $(x=0)$ is contact with the upstream reservoir directly.

(3) $m[p(L, t)]=m\left[p_{2}(\mathrm{t})\right]$, for $t \geq 0$. This states that the downstream end of the sample $(x=L)$ is contact with the downstream reservoir directly.

(4) $\frac{d\left[m\left(p_{1}\right)\right]}{d t}=\frac{a k}{\varphi \mu L\left(C_{g}+C_{f}\right)} \frac{\partial[m(p)]}{\partial x}$, where $a$ is the ratio of the sample pore volume $\left(V_{p}\right)$ over the upstream reservoir volume $\left(V_{1}\right)$; for $x=0$, and $t \geq 0$. This expresses mass conservation at the upstream end of the sample.

(5) $\frac{d\left[m\left(p_{2}\right)\right]}{d t}=-\frac{b k}{\varphi \mu L\left(C_{g}+C_{f}\right)} \frac{\partial[m(p)]}{\partial x}$, where $b$ is the ratio of the sample pore volume $\left(V_{p}\right)$ over the downstream reservoir volume $\left(V_{2}\right)$; for $x=L$, and $t \geq 0$. This expresses mass conservation at the downstream end of the sample.

Hsieh et al. [19] and Dicker and Smits [20] gave the exact solution to the diffusivity equation (Eq. 1) for the pressure in the downstream reservoir, which is

$$
\frac{m\left[p_{2}(t)\right]-m\left[p_{2}(0)\right]}{m\left[p_{1}(0)\right]-m\left[p_{2}(0)\right]}=\frac{b}{a+b+a b}+2 \sum_{m=1}^{\infty} \frac{e^{-t_{D} \theta_{m}^{2}}\left(a b^{2}-b \theta_{m}^{2}\right)}{\left[\theta_{m}^{4}+\theta_{m}^{2}\left(a+a^{2}+b+b^{2}\right)+a b(a+b+a b)\right] \cos \theta_{m}} \cdots \cdots \cdots \cdots \cdots \cdots \cdot \cdots \cdot \cdots \cdots \cdots \cdot
$$

where $\theta_{m}$ can be calculated from the following equation

$$
\tan \theta=\frac{(a+b) \theta}{\theta^{2}-a b}
$$

In Eq.2, the dimensionless time, $t_{D}$, is defined as:

$$
t_{D}=\frac{k t}{\varphi \mu\left(C_{g}+C_{f}\right) L^{2}}
$$


By careful observation, we find that $\mu z$ can be treated as constant in the range of pressure less than 2000 psi [21], which is the pressure conditions used in this study. According to $m(p)=\int_{p_{b}}^{p} \frac{2 p}{\mu z} d p$, the left-hand-side (LHS) of Eq. 2 can be written as

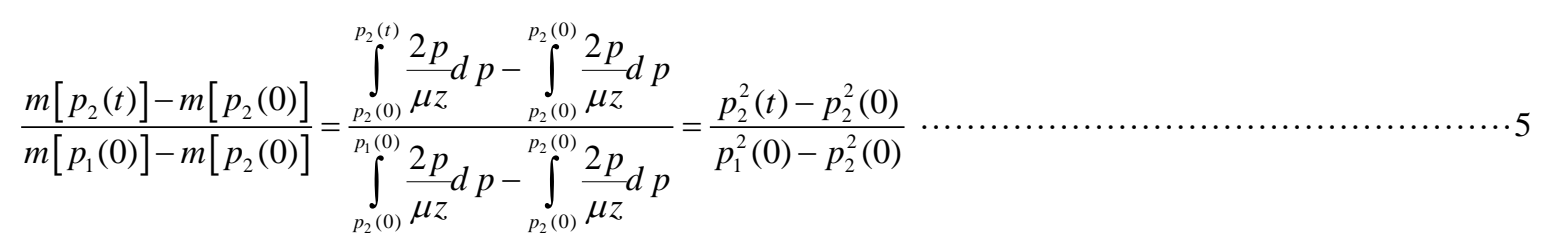

Next, we simplify the right-hand-side (RHS) of Eq. 2. The upstream pressure $p_{1}$ is invariant throughout the test, which implies that the upstream volume $V_{1}$ becomes infinity, so the ratio of the sample pore volume to the upstream volume $\left(a=\frac{V_{p}}{V_{1}}\right)$ can be considered as zero. Substituting a as zero and Eq.5 into Eq.2, we obtain:

$$
\frac{P_{2}^{2}(t)-p_{2}^{2}(0)}{p_{1}^{2}(0)-p_{2}^{2}(0)}=1+2 \sum_{m=1}^{\infty} \frac{e^{-t_{D} \theta_{m}^{2}}\left(-b \theta_{m}^{2}\right)}{\left[\theta_{m}^{4}+\theta_{m}^{2}\left(b+b^{2}\right)\right] \cos \theta_{m}},
$$

which can be written as

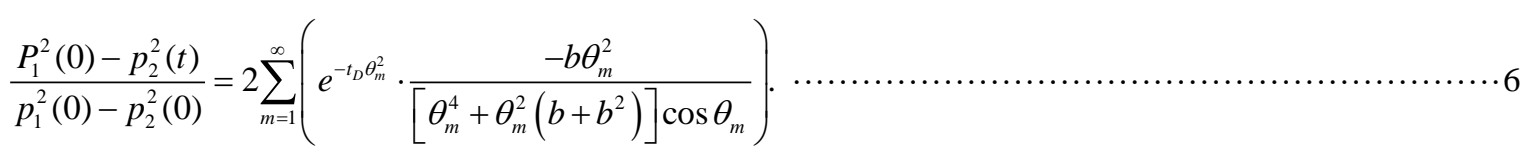

For $a=0$, Eq.3 becomes

$$
\tan \theta=\frac{b}{\theta}
$$

which can be written in the following format $\frac{\sin \theta}{\cos \theta}=\frac{b}{\theta}$ that leads to $\cos \theta=\sqrt{\frac{\theta^{2}}{\theta^{2}+b^{2}}}$. This equation contains infinite solutions of $\theta_{m}$, and the values of the solutions increase monotonically. Thus $\cos \theta_{m}=(-1)^{m-1} \frac{\theta_{m}}{\sqrt{\theta_{m}^{2}+b^{2}}}$. Inserting it into Eq.6, we obtain

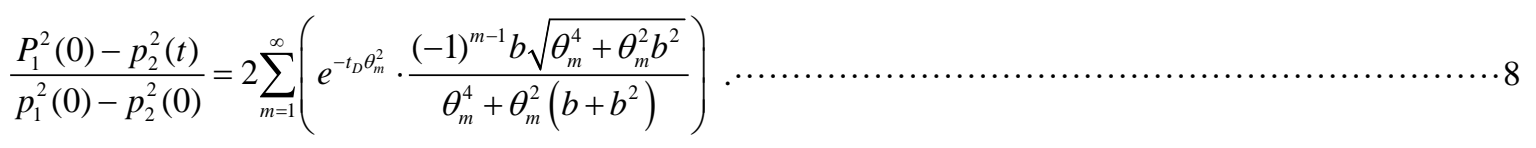


Dicker and Smits [20] mentioned that Eq. 8 is not fully single exponentially decreasing because the volume of upstream reservoir is much larger than that of downstream reservoir. But they indicated that the experiment under this condition was rapid. In addition, a single exponential equation fitted very well with the downstream reservoir pressure buildup curve if a right interval was selected. Thus, we simplified Eq. 8 to

$\frac{P_{1}^{2}(0)-p_{2}^{2}(t)}{p_{1}^{2}(0)-p_{2}^{2}(0)}=e^{-t_{D} \theta_{1}^{2}} \cdot\left(2 \frac{b \sqrt{\theta_{1}^{4}+\theta_{1}^{2} b^{2}}}{\theta_{1}^{4}+\theta_{1}^{2}\left(b+b^{2}\right)}\right)$

Let $\Delta p(t)=p_{1}^{2}(0)-p_{2}^{2}(t)$, we get $\frac{\Delta p(t)}{\Delta p(0)}=\left(2 \frac{b \sqrt{\theta_{1}^{4}+\theta_{1}^{2} b^{2}}}{\theta_{1}^{4}+\theta_{1}^{2}\left(b+b^{2}\right)}\right) \cdot e^{-t_{D} \theta_{1}^{2}}$, which can be written as

$\Delta p(t)=\left(2 \Delta p(0) \cdot \frac{b \sqrt{\theta_{1}^{4}+\theta_{1}^{2} b^{2}}}{\theta_{1}^{4}+\theta_{1}^{2}\left(b+b^{2}\right)}\right) \cdot e^{-t_{D} \theta_{1}^{2}}$

Taking the natural log of Eq. 9 yields

$\ln [\Delta p(t)]=\ln \left(2 \Delta p(0) \cdot \frac{b \sqrt{\theta_{1}^{4}+\theta_{1}^{2} b^{2}}}{\theta_{1}^{4}+\theta_{1}^{2}\left(b+b^{2}\right)}\right)+\left(-t_{D} \theta_{1}^{2}\right)$

Substituting $t_{D}$ of Eq.4 into Eq.10, we get

$\ln [\Delta p(t)]=\ln \left(2 \Delta p(0) \cdot \frac{b \sqrt{\theta_{1}^{4}+\theta_{1}^{2} b^{2}}}{\theta_{1}^{4}+\theta_{1}^{2}\left(b+b^{2}\right)}\right)-\frac{\theta_{1}^{2} k}{\varphi \mu\left(C_{g}+C_{f}\right) L^{2}} \cdot t$

Based on Eq. 11, we plot a graph of the pressure difference in a logarithm as a function of time (Fig. 4), and obtain

the value of $\frac{-\theta_{1}^{2} k}{\varphi \mu\left(C_{g}+C_{f}\right) L^{2}}$ as the slope of the linear fitting line, $s$.

$k=\frac{\varphi \mu\left(C_{g}+C_{f}\right) L^{2}}{\theta_{1}^{2}} \cdot(-s)$

or

$C_{f}=\frac{-k \theta_{1}^{2}}{\varphi \mu L^{2} s}-C_{g}$ 
Using the Taylor series expansion, $\tan \theta \approx \theta+\frac{\theta^{3}}{3}$, and combining with Eq.7, we obtain $\theta_{1}+\frac{\theta_{1}^{3}}{3}=\frac{b}{\theta_{1}}$, from which we have:

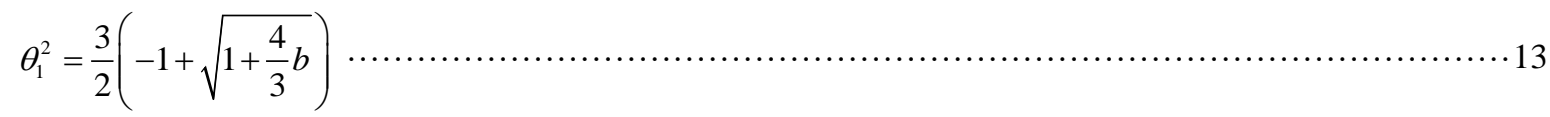

Substituting Eq.13 and $b=\frac{V_{p}}{V_{2}}=\frac{\varphi A L}{V_{2}}=\frac{\varphi \pi D^{2} L}{4 V_{2}}$ into Eq. 12, we obtain:

$$
\begin{aligned}
C_{f} & =\frac{-3 k\left(-1+\sqrt{1+\frac{4}{3} b}\right)}{2 \varphi \mu L^{2} s}-C_{g} \\
& =\frac{3 k\left(1-\sqrt{1+\frac{\varphi \pi D^{2} L}{3 V_{2}}}\right)}{2 \varphi \mu L^{2} s}-C_{g},
\end{aligned}
$$

\section{Sample and Equipment}

Middle Bakken core samples were chosen as the test specimens in this study. The core plugs were cylindrical with a dimension of one inch in diameter and two inches in length.

First the cylindrical core plug was covered with copper sheeting in order to make a gas-tight seal on the cylindrical wall of the sample and to apply radial confining pressure. Then the core plug was mounted in a sample holder with flexible rubber sleeves at both ends of the plug (Fig. 5). Last, the core holder was put into a vessel flooded with mineral oil, in which the sample could be hydrostatically compressed by applying pressure by hydraulic means. To minimize the volume of the downstream reservoir, a small pocket is implemented inside the downstream end-cap. The volume of downstream reservoir is 0.63 cubic centimeters.

The equipment used to perform the experiments is AutoLab-1500, which was made by New England Research Inc. Fig. 6 presents a conceptual diagram of test facility. One temperature transducer (TT1) was used to measure the temperature. Four pressure transducers, PT1, PT2, PT3, and PT4, were used to measure: upstream reservoir 
pressure, confining pressure at the flank of the core, downstream reservoir pressure, and axial pressure at the ends of the core, respectively.

\section{Measurement Procedure}

The experiment was conducted under room temperature, which was $25{ }^{\circ} \mathrm{C}$. The determination of the permeability was a three-step process, namely installing the core plug into AutoLab-1500, running the test, and analyzing the resultant data.

1) Installing the core plug into AutoLab-1500

First, the core holder was placed into the vessel, then the vessel was filled with mineral oil and the confining pressure was increased to the desired level $\left(p_{c}\right), 15 \mathrm{MPa}$ (2175 psi). The valve between the core plug and the upstream reservoir was closed. Nitrogen gas was used to fill the upstream reservoir, and the upstream pressure was increased to a desired level $\left(p_{1}\right)$, 8.5 MPa (1232 psi). The downstream pressure was atmospheric pressure. Notice that the confining pressure must be greater than the upstream pressure.

\section{2) Running the test}

The starting time was recorded when the inlet valve between the core plug and the upstream reservoir was opened (Fig. 2). During the whole test, the upstream and the confining pressures were kept constant. The pressures were monitored and recorded at both upstream and downstream ends of the sample (Fig. 2).

\section{3) Analyzing the resultant data}

To obtain the compressibility by downstream reservoir pressure build-up method, firstly we calculated the pressure difference in a logarithm scale from equation: $\ln [\Delta p(t)]=\ln \left[p_{1}^{2}(0)-p_{2}{ }^{2}(t)\right]$, and then from the plot (see Fig. 4), we obtained the slope, s. Finally, Eq.14 was used to calculate the compressibility of the rock.

\section{Results and Discussions}


The compressibilities of six Bakken core plugs were calculated by the proposed model (Table 1). Because of the low to extremely low permeability of Bakken sample, an oscillating pulse method was used to measure the permeabilities of these plugs. The oscillating pulse method is a commercial technique provided by the Autolab-1500 system from New England Research Inc. In order to validate the proposed method, the compressibilities were also measured by triaxial stress experiment (Table 1). The comparison indicates that the compressibilities from the proposed method are close to those from the triaxial stress experiment. Therefore, the proposed method provides a reliable way to determine the compressibility.

Because the cost to measure rock compressibility can be high and time consuming, the proposed model provides a way to estimate rock compressibility from permeability experimental data in case direct compressibility measurement is not available. In case the direct compressibility measurement is available, the model can serve as a quality control tool.

The accuracy of the proposed model depends on the accuracies of rock porosity, permeability, core length, and gas compressibilities. A good estimation of gas viscosity and permeability at measured conditions is also important. This proposed method is not appropriate for the fractured sample due to its high heterogeneity. In this situation, the assumption of homogeneous cannot be met.

\section{Acknowledgment}

The authors are grateful to The Petroleum Engineering Department at the University of North Dakota. This research is supported in part by the U.S. Department of Energy (DOE) under award number DE-FC26-08NT0005643 and North Dakota EPSCoR Program under award number EPS-0814442. The authors would like to thank the Wilson M. Laird Core and Sample Library and the North Dakota Geological Survey for providing core samples for this study.

\section{Nomenclature}

A : $\quad$ area of the cross section of the core plug 


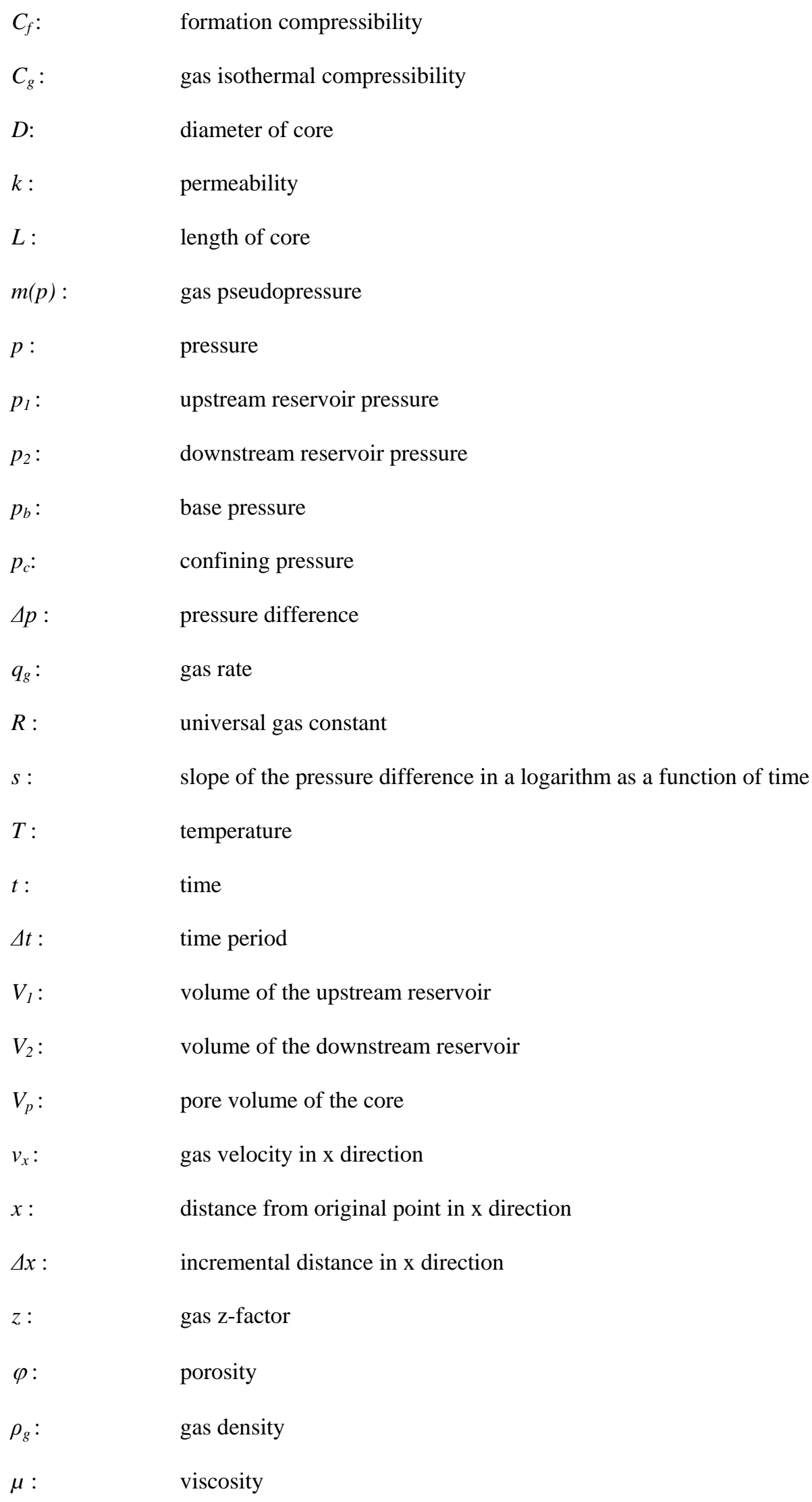


$\mu_{g}: \quad$ gas viscosity

\section{References}

1. Carpenter, C. R. and Spencer, G. R. 1940. Measurements of Compressibility of Consolidated Oil-Bearing Sandstones, Bureau of Mines RI 3540, (Oct. 1940).

2. Hall, H. N. 1953. Compressibility of Reservoir Rocks, Journal of Petroleum Technology, Vol. 5, No. 1, p. $17-19$.

3. Fatt, I. 1958. Pore Volume Compressibilities of Sandstone Reservoir Rocks, Journal of Petroleum Technology, Vol. 10, No. 3, p. 64-66.

4. Van der Knaap, W. 1959. Nonlinear Behavior of Elastic Porous Media, Petroleum Transactions, AIME, Vol. 216, p. 179-187.

5. Harville, D. W., and Hawkins, M. F. 1969. Rock Compressibility and Failure as Reservoir Mechanisms in Geopressured Gas Reservoirs, Journal of Petroleum Technology, Vol. 21, No. 12, p. 1528-1530.

6. Greenwald, R. F. and Somerton, W. H. 1981a. Pore Volume Compressibility Data for Bandera, Berea, and Boise Sandstones, SPE 9746-MS.

7. Greenwald, R. F. and Somerton, W. H. 1981b. A Theoretical Model For Determination of Pore Volume Compressibilities of Consolidated Sandstones, SPE 10076-MS, SPE Annual Technical Conference and Exhibition, 4-7 October 1981, San Antonio, Texas.

8. Zimmerman R. W., Somerton, W. H., and King, M. S. 1986. Compressibility of Porous Rocks, Journal of Geophysical Research, Vol. 91, No. B12, p. 12765-12777. 
9. Poston, S.W. and Chen, H.Y. 1987. The Simultaneous Determination of Formation Compressibility and Gas-in-Place in Abnormally Pressured Reservoirs, SPE 16227, SPE Production Operations Symposium, 810 March 1987, Oklahoma City, Oklahoma.

10. Chalaturnyk, R. J. and Scott, J. D. 1992. Evaluation Of Reservoir Properties From Geomechanical Tests, Journal of Canadian Petroleum Technology, Vol. 31, No. 5, p. 31-40.

11. Khatchikian, A. 1996. Deriving Reservoir Pore-Volume Compressibility From Well Logs, SPE Advanced Technology Series, Vol. 4, No. 1, p. 14-20.

12. Yildiz, T. 1998. Prediction of In-Situ Formation Compressibility from Production Data, SPE 47379-MS, SPE/ISRM Rock Mechanics in Petroleum Engineering, 8-10 July 1998, Trondheim, Norway.

13. Macini, P. and Mesini, E. 1998. Static and Dynamic Reservoir Rock Compressibility at High Pressure, SPE 49549-MS, Abu Dhabi International Petroleum Exhibition and Conference, 11-14 November 1998, Abu Dhabi, United Arab Emirates.

14. Marchina, P., Brousse, A., Fontaine, J., Dano, C., and Alonso, C. 2004. In-situ Measurement of Rock Compressibility in a Heavy Oil Reservoir, SPE 86940-MS, SPE International Thermal Operations and Heavy Oil Symposium and Western Regional Meeting, 16-18 March 2004, Bakersfield, California.

15. Li, C., Chen, X., and Du, Z. 2004. A New Relationship of Rock Compressibility with Porosity, SPE 88464MS, SPE Asia Pacific Oil and Gas Conference and Exhibition, 18-20 October 2004, Perth, Australia.

16. Suman, A. 2009. Uncertainties in Rock Pore Compressibility and Effects on Seismic History Matching, Master Thesis, Stanford University. 
17. Ghanbarnezhad Moghanloo, R. and Javadpour, F. 2014. Applying Method of Characteristics to Determine Pressure Distribution in 1D Shale-Gas Samples. SPE Journal, Vol. 19, Issue 03, p. 361-372 doi:10.2118/168218-PA.

18. Al-Hussainy, R., Ramey, H.J., and Crawford, P.B. 1966. The Flow of Real Gases through Porous Media. Journal of Petroleum Technology, p. 624-636.

19. Hsieh, P. A., Tracy, J. V., Neuzil, C. E., Bredehoeft, J. D., and Silliman, S. E. (1981). A transient laboratory method for determining the hydraulic properties of 'tight' rocks-I. Theory. International Journal of Rock Mechanics and Mining Sciences \& Geomechanics Abstracts, 18, P245-252.

20. Dicker, A. I., and Smits, R. M. (1988). A Practical Approach for Determining Permeability from Laboratory Pressure-Pulse Decay Measurements. SPE 17578, P285-292.

21. Craft, B.C., Hawkins, M., Terry, R.E. 1991. Applied Petroleum Reservoir Engineering, $2^{\text {nd }}$ edition, Prentice Hall Professional, Inc., Englewood Cliffs, NJ, 1991, page 221. 


\begin{tabular}{|l|l|l|l|l|l|l|l|}
\hline \multicolumn{6}{|c|}{ Table 1. Compressibility measured by use of pressure buildup in permeability experiment } \\
\hline & unit & Core 1 & Core 2 & Core 3 & Core 4 & Core 5 & Core 6 \\
\hline$L$ & in & 2.7780 & 2.7224 & 2.7008 & 2.3882 & 2.6992 & 2.5819 \\
\hline$\varphi$ & in & 1.0311 & 1.0394 & 1.0327 & 1.0323 & 1.0291 & 1.0315 \\
\hline$C_{g}$ & fraction & 0.044 & 0.045 & 0.032 & 0.035 & 0.036 & 0.054 \\
\hline$\mu$ & $10^{-6} / \mathrm{psi}$ & 125 & 125 & 125 & 125 & 125 & 125 \\
\hline$V_{2}$ & $\mathrm{cp}$ & 0.0474 & 0.0474 & 0.0474 & 0.0474 & 0.0474 & 0.0474 \\
\hline$s$ & $\mathrm{ft}$ & $2.22 \mathrm{E}-05$ & $2.22 \mathrm{E}-05$ & $2.22 \mathrm{E}-05$ & $2.22 \mathrm{E}-05$ & $2.22 \mathrm{E}-05$ & $2.22 \mathrm{E}-05$ \\
\hline$k$ & $\mathrm{Ln}\left(\mathrm{psi}{ }^{2}\right) / \mathrm{h}$ & -2.4588 & -1.3644 & -1.818 & -1.2528 & -2.4228 & -78.12 \\
\hline$C_{f}$ (Proposed method) & $\mathrm{HD}$ & 0.26 & 0.14 & 0.18 & 0.12 & 0.24 & 8.0 \\
\hline$C_{f}$ (Triaxial stress method) & $10^{-6} / \mathrm{psi}$ & 5.11 & 6.21 & 2.95 & 5.47 & 6.90 & 5.55 \\
\hline Relative error & $\%$ & 10.35 & -8.63 & -4.07 & -7.73 & 5.13 & 4.00 \\
\hline
\end{tabular}




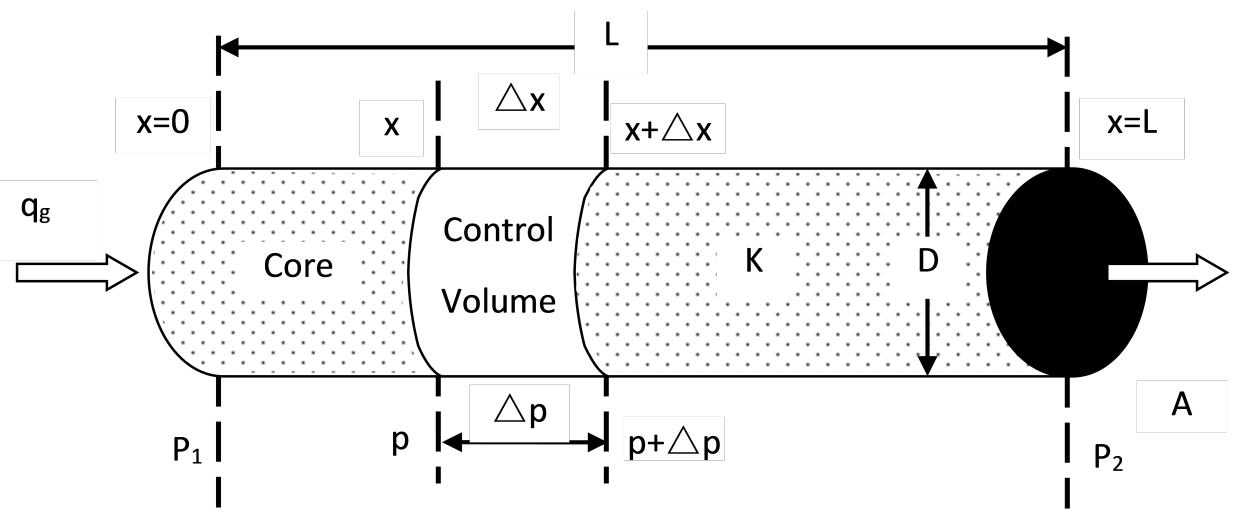

Figure 1: Gas flows through core during permeability measurement

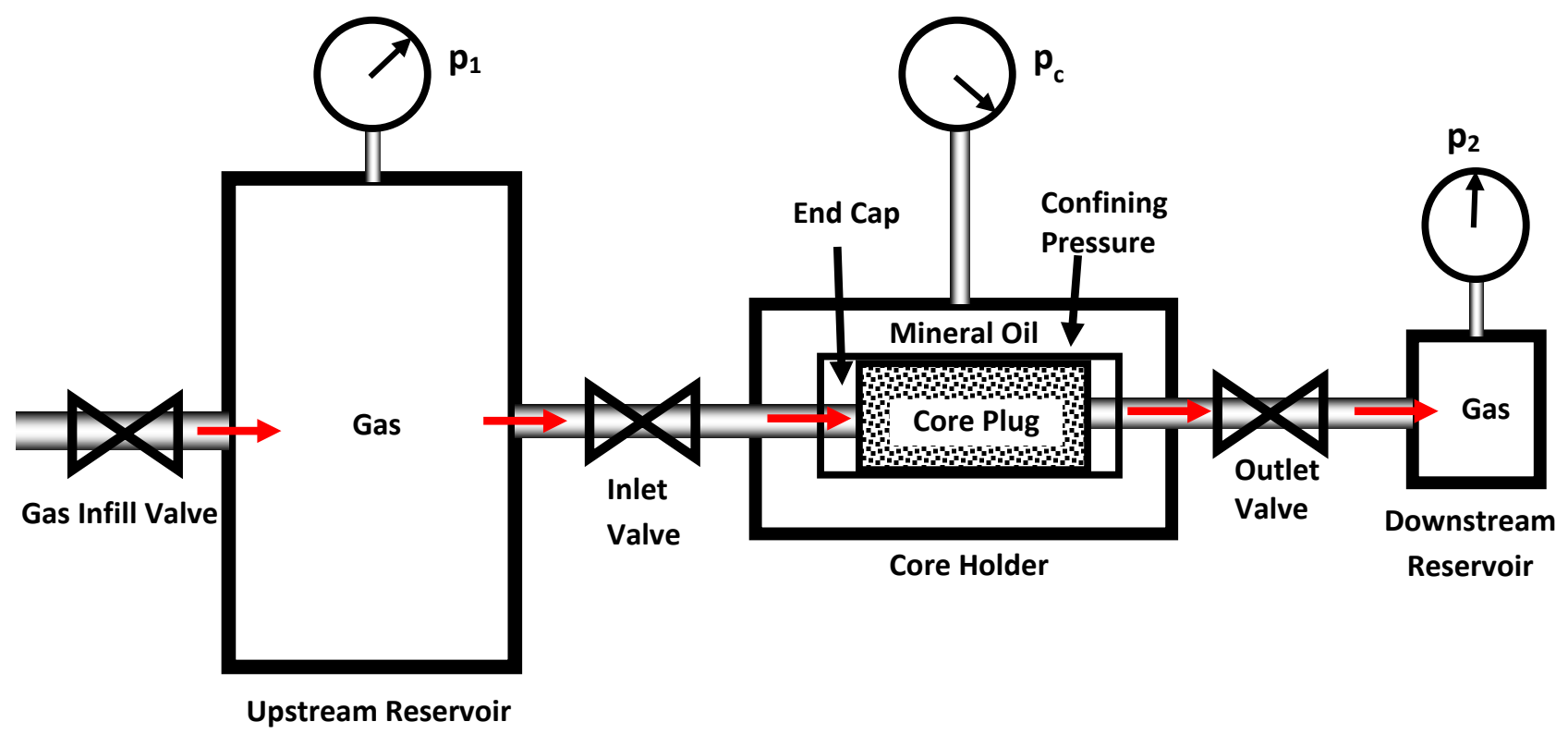

Figure 2: Schematic of experimental setup to estimate rock compressibility 


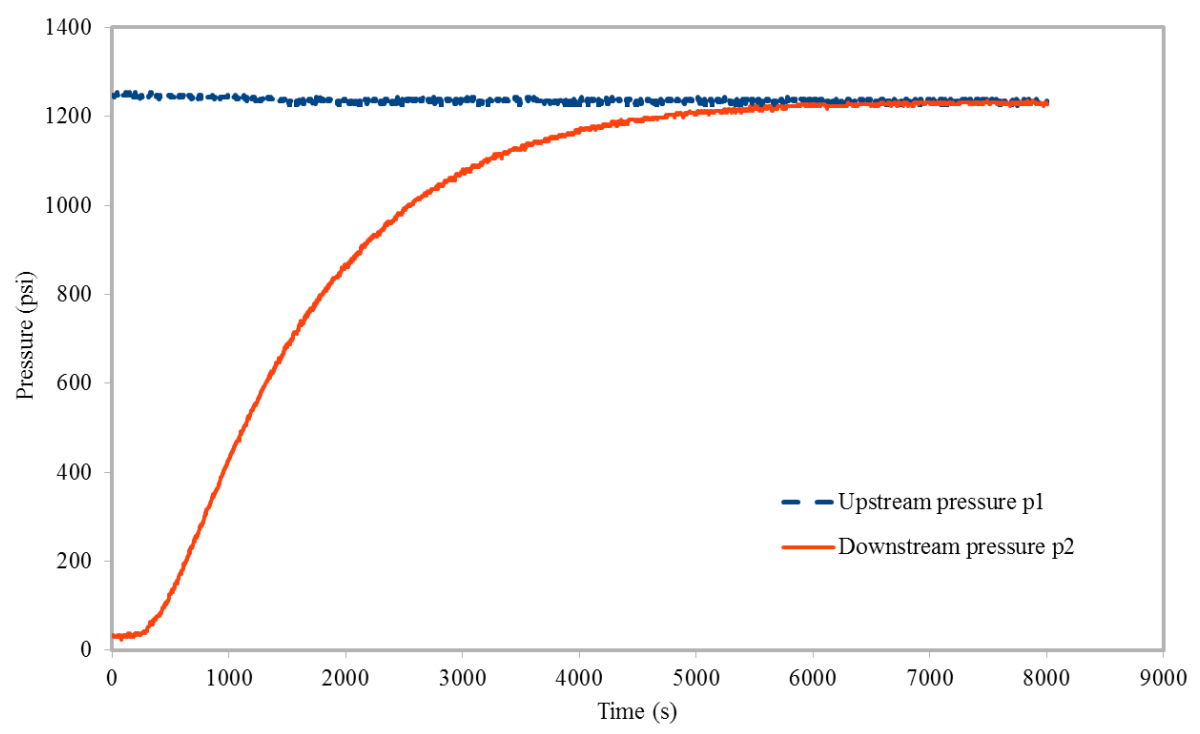

Figure 3: Changes of the upstream and downstream pressures during experiment

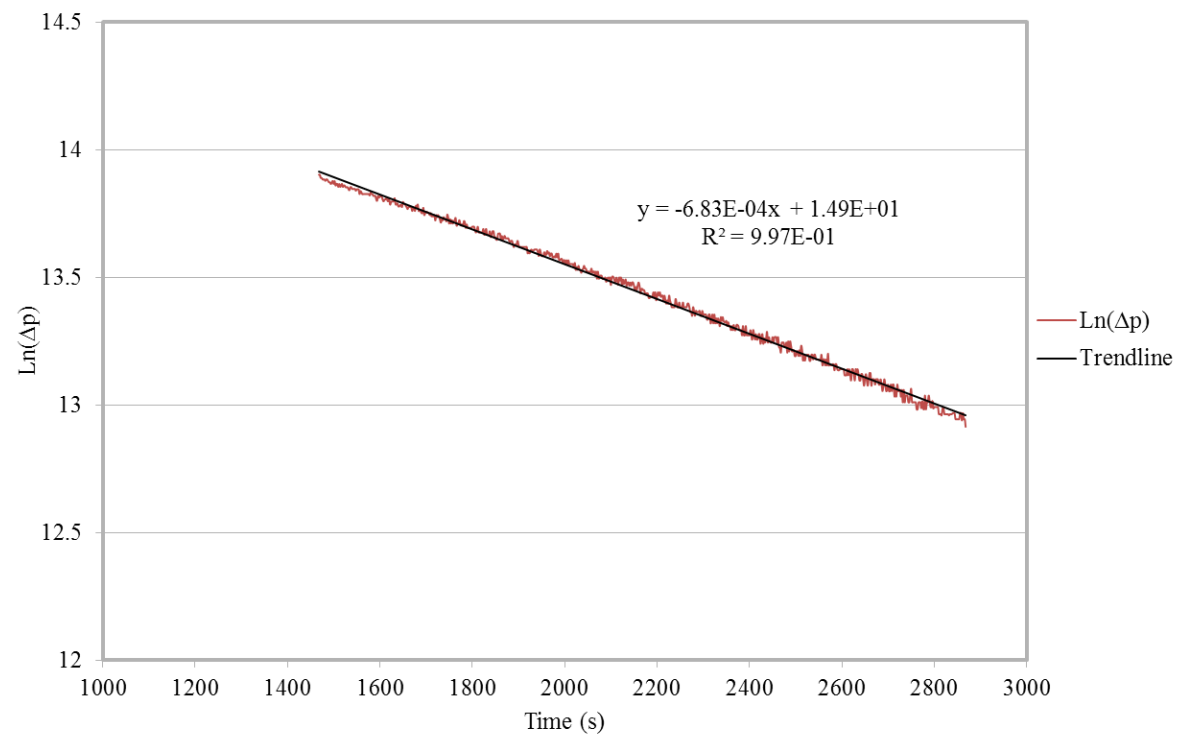

Figure 4: $\ln (\Delta p)$ vs. time plot for a core sample. 


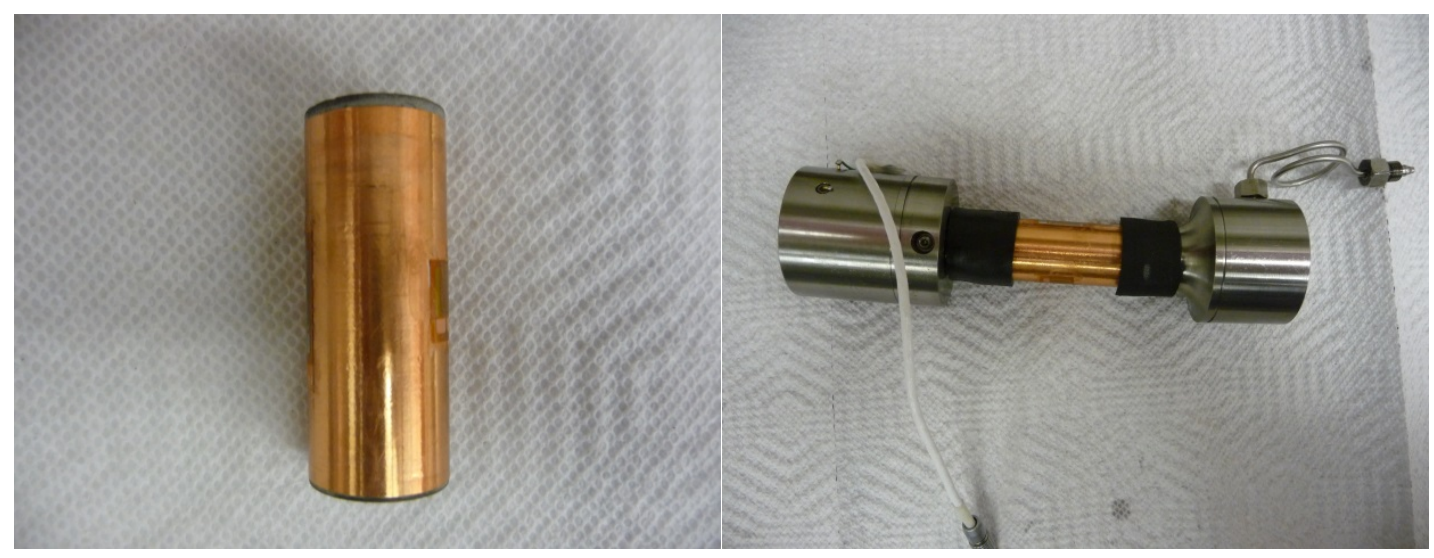

Figure 5: Images of core and core holder for low permeability test system

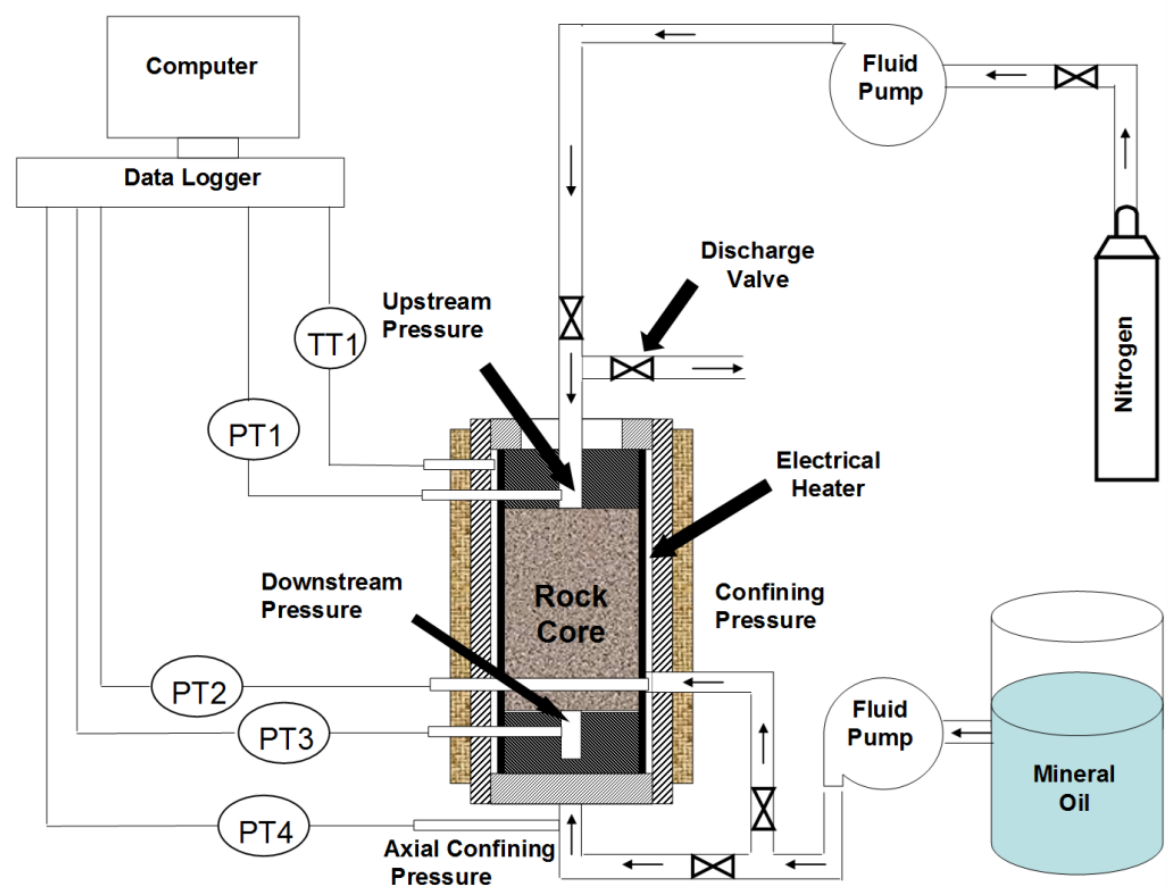

Figure 6: Experimental setup for permeability measurement under stresses 